\title{
Thyroidectomy with Ligasure Versus Traditional Thyroidectomy
}

H.R.Mosalam, N.A.Zaher, M.I.Abou Rizk and A.A.Elgohary

General Surgery Dept., Faculty of Medicine, Benha Univ., Benha, Egypt

E-Mail: Elgohary@gmail.com

\begin{abstract}
New surgical devices have been developed to improve hemostatic control and vascular closure in thyroid surgery.This study aims to evaluate how safe and feasible is the ligaSure for thyroidectomy and to compare its results with the conventional thyroidectomy technique. This examination is a relative forthcoming randomized control one intended for patients having amiable thyroid sores. We incorporated a sum of 20 cases who were isolated into 2 equivalent gatherings; gathering (An) included 10 cases who experienced the traditional method, and the other one (B) incorporated the other 10 cases who experienced the activity with LigaSure. All cases were exposed to finish history taking, exhaustive physical assessment, and routine research facility and radiological examinations before surgery.Patient qualities didn't vary fundamentally between the two gatherings. The traditional methodology had a fundamentally drawn out employable time when contrasted with LigaSure (75.38 versus 47.13 minutes individually $-\mathrm{p}<0.001)$. No intraoperative difficulties were experienced in our investigation. No distinction was recognized with respect to post-usable agony between the two investigation gatherings $(\mathrm{p}=0.97)$. One case $(10 \%)$ in the ordinary gathering was convoluted by hematoma and was overseen by means of careful investigation, while no cases built up that entanglement in the other gathering $(\mathrm{p}=0.372)$. Post-employable intricacies were not fundamentally extraordinary between the two gatherings. It is apparent that ligaSure isn't just a sheltered technique for hemostasis during thyroid medical procedure like the regular methodology, however it likewise assists with playing out the medical procedure in an essentially shorter employable time.
\end{abstract}

Keywords: Thyroidectomy, LigaSure, Operative time.

\section{Introduction}

The thyroid organ and its hormones assume multifaceted jobs in organ improvement and in the homeostatic control of basic physiological systems, for example, body development and vitality consumption in all vertebrates [1].

The thyroid organ is a profoundly vascular endocrine organ that is provided by the unrivaled, substandard thyroid veins, thyroid ima course, tracheal and esophageal branches [2].

Safe thyroid medical procedure requires fastidious hemostasis and cautious control of dying. The principle wellsprings of draining are harmed thyroid vessels and thyroid parenchymal dying [3].

Drain (intra-or postoperative) may cause a few intricacies as seromas as well as hematomas, every so often answerable for conceivably deadly asphyxia. Along these lines, hemostasis is a basic factor deciding likewise the recurrence of different issues, just as the prolongation of the usable time, in this way expanding the length of clinic remain and costs [4].

Numerous strategies intended to keep up careful hemostasis are by and by utilized: ligation and stitching (strings, cuts, staplers), coagulation (monopolar and bipolar electrocoagulation), ultrasonic coagulation (Ultracision, Harmonic Scalpel@; Ethicon Endo-Surgery, Cincinnati, OH), electroligation fixing (LigaSure® Vessel Sealing System; Valleylab, Boulder, CO, USA) [3].

The LigaSure ${ }^{\mathrm{TM}}$ Vessel Sealing System (Valley Lab, Boulder, CO) is another hemostatic gadget. It is a bipolar electrosurgical gadget, fixing vessels up to $7 \mathrm{~mm}$ in breadth, by denaturing collagen and elastin inside vessel divider and encompassing connective tissue. The LigaSure has a diminished vitality spread profile $(<2$ $\mathrm{mm}$ ) when contrasted and unipolar searing, with a potential diminished danger of injury to neighboring structures [5].

The danger of draining doesn't seem to increment with LigaSure use, and a few reports have even demonstrated decreased usable blood misfortune just as working time [6].

\section{Patients and methods}

This examination is a near imminent randomized control one intended for patients having amiable thyroid injuries who were treated by thyroidectomy between January 2019 and July 2019. The essential result of this investigation was to evaluate the length of activity and the occurrence of post-usable dying. Different results included medical clinic remain, post-employable torment, hypocalcemia, and careful site contamination.

We incorporated an aggregate of twenty patients who experienced thyroidectomy during that period. Cases with straightforward multinodular goiter, poisonous goiter, or singular thyroid knob were incorporated. By the by, cases with enormous, repetitive, or threatening goiters, notwithstanding past neck radiation were barred from the present investigation.

Our cases were separated into 2 equivalent gatherings; gathering (An) included 10 cases who experienced the regular methodology, while gathering (B) incorporated the staying 10 cases who experienced thyroidectomy by LigaSure.

All cases were exposed to finish history taking, intensive physical assessment, and routine preemployable research center examinations including thyroid profile. In addition, neck ultrasonography was requested for all cases while fine needle yearning cytology (FNAC) was likewise requested when demonstrated. All cases indicated various degrees of thyroid status so they were medicinally figured out how to come to the euthyroid state before activity. 


\section{Surgical technique}

In the ordinary strategy, the thyroid was drawn nearer by means of a low transverse neckline entry point. After height of the platysma fold, superiorly and poorly, the lash muscles were partitioned in the midline and raised pointedly from the hidden thyroid organ. The dismemberment of the thyroid started with ligation division of the center thyroid vein. Consideration was coordinated toward representation of the sub-par and unrivaled parathyroid organs. The second rate thyroid vein was ligated after recognizable proof of the intermittent laryngeal nerve, the unrivaled post vessels were ligated Fig (1), and the thyroid was raised from the pretracheal sash. The dismemberment was conveyed over the midline and finished by assembling the isthmus and pyramidal projection of the thyroid Fig (2).

In LigaSure method, we utilized a Valleylab framework (LigaSure Bipolar Vessel Sealing System, LigaSure, Valleylab, Boulder, CO, USA, Tyco Healthcare Group L.P.) with a generator (LigaSure vessel fixing generator, ForceTriad vitality stage), just as the LigaSure Precise instrument, were utilized for the LigaSure medical procedures. The careful method was pretty much like the traditional one yet LigaSure was utilized rather than stitch ligation and division Fig (3).

All patients were conveyed to the recuperation room postoperatively. From that point forward, they were moved to the inward ward. Post-employable agony was surveyed by means of visual simple score VAS [7]. In addition, post-usable complexities, particularly postemployable dying, were likewise checked. Except if entanglements were envisioned, patients were permitted to be released as a rule on the second post-employable day.

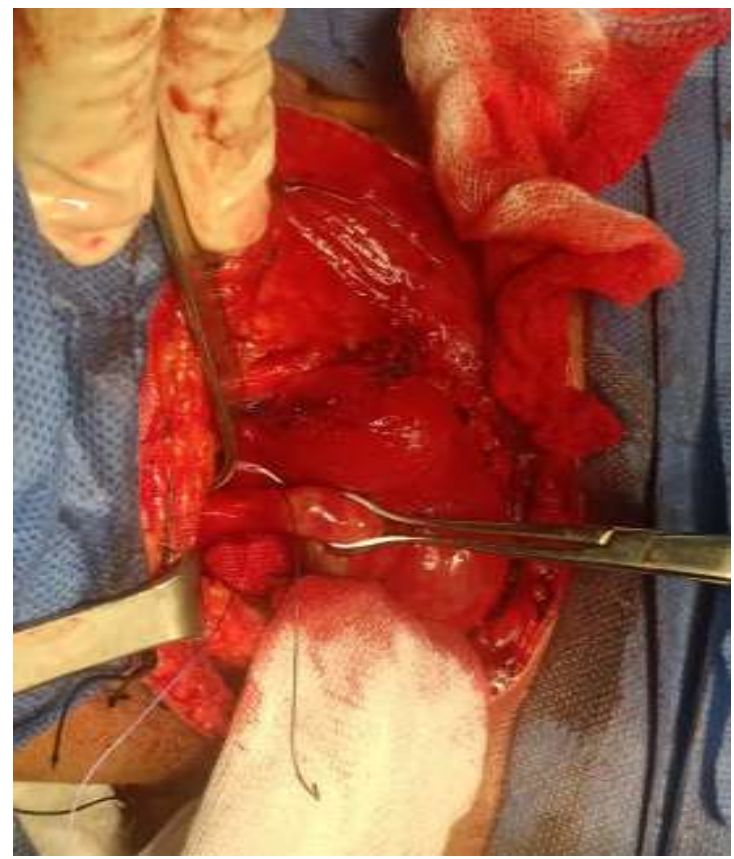

Fig (1) Ligation of superior thyroid vessels (Rt) by conventional method.

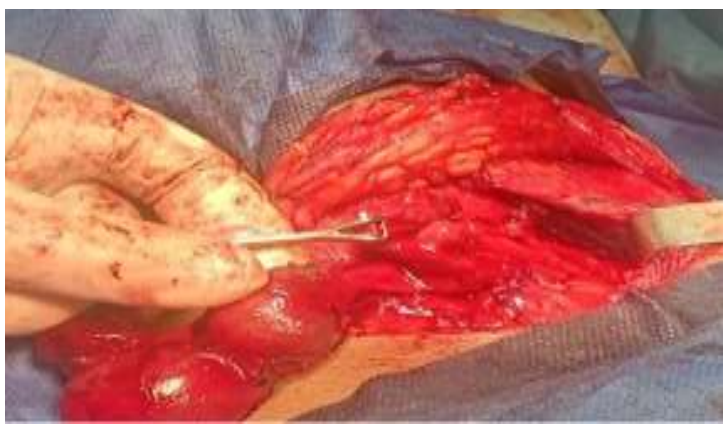

Fig (2) Dissection across the midline and mobilization of the isthmus of the thyroid.

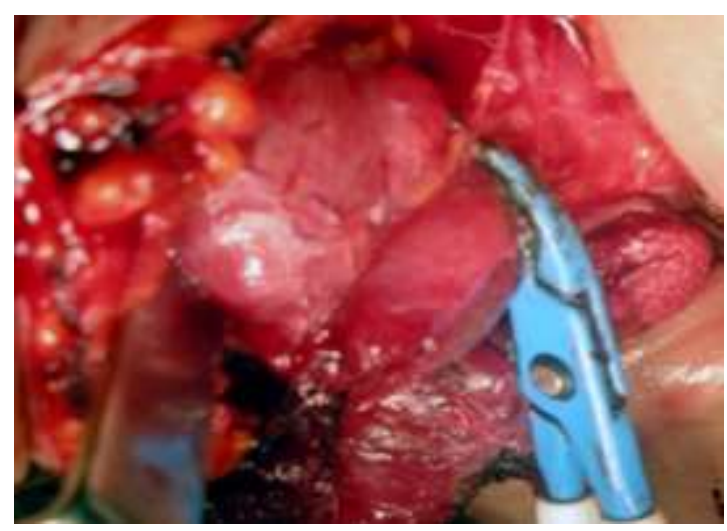

Fig (3) LigaSure in the inferior lobe.

\section{Ethical consideration}

A written informed consent was obtained from all patients before the operation after describing and explaining the operative and postoperative details and complications of each approach.

\subsection{Statistical analysis}

Statistical analysis of the data in this study was performed using SPSS software, version 20 (Chicago, IL). Descriptive data was expressed as means with standard deviation or medians with ranges according to data distribution. Categorical variables were described using frequency distributions. Independent sample t-test was used to detect differences in the means of continuous variables and Chi-square test was used in cases with low expected frequencies. $\mathrm{P}$ values $<0.05$ were considered significant.

\section{Results}

Regarding demographics, the mean age of the included cases was 48.34 and 49.26 years for group A and $\mathrm{B}$ respectively. Female gender occupied $70 \%$ (7 cases) and $80 \%$ (8 cases) of the study cases for both groups respectively. Regarding comorbidities, two cases had a history of diabetes mellitus in every group while hypertension was present 2 cases $(20 \%)$ and 1 case $(10 \%)$ in both groups in order of speech. 
Table (1) Preoperative data.

\begin{tabular}{lccc}
\hline & $\begin{array}{c}\text { Group A }(\mathbf{n}= \\
\mathbf{1 0})\end{array}$ & Group B (n = 10) & P value \\
\hline Age & $48.34 \pm 12.84$ & $49.26 \pm 11.32$ & 0.645 \\
Gender & & & \\
-Male & $3(30 \%)$ & $2(20 \%)$ & 0.327 \\
-Female & $7(70 \%)$ & $8(80 \%)$ & \\
\hline Comorbidities & & & 0.208 \\
-DM & $2(20 \%)$ & $2(20 \%)$ & \\
-Hypertension & $2(20 \%)$ & $1(10 \%)$ & \\
-IHD & $1(10 \%)$ & $1(5 \%)$ & 0.418 \\
Diagnosis & & $3(30 \%)$ & \\
-Follicular lesion & $3(30 \%)$ & $6(60 \%)$ & \\
-SMNG & $5(50 \%)$ & $1(10 \%)$ & \\
-Toxic goiter & $2(20 \%)$ & & \\
\hline
\end{tabular}

In group $\mathrm{B}$, the cases were divided equally between two operations; total and hemi-thyroidectomy, that were performed in 10 cases $(50 \%)$ for each. On the other hand, hemithyroidectomy was performed in 4 cases (40\%) while 6 cases $(60 \%)$ underwent total thyroidectomy in group A. No intraoperative complications were encountered in our study. Table (2) illustrates these data.

Table (2) Operative data.

\begin{tabular}{lccc}
\hline & $\begin{array}{c}\text { Group A }(\mathbf{n}= \\
\mathbf{1 0})\end{array}$ & Group B $(\mathbf{n}=\mathbf{1 0})$ & P value \\
\hline Type of operation & & & 0.386 \\
-Total thyroidectomy & $6(60 \%)$ & $5(50 \%)$ & \\
-Hemithyroidectomy & $4(40 \%)$ & $5(50 \%)$ & \\
Operative time (min) & $75.38 \pm 8.76$ & $47.13 \pm 2.84$ & $<0.001$ \\
\hline
\end{tabular}

Age and comorbidities had no significantly differences between the study groups $(p>0.05)$. The conventional approach had a significantly prolonged operative time when compared to LigaSure (75.38 vs. 47.13 minutes respectively $-\mathrm{p}<0.001$ ).

No difference was detected regarding post-operative pain between the two study groups $(\mathrm{p}=0.97)$. One case $(10 \%)$ in group A was complicated by hematoma and was managed via surgical exploration, while no cases developed that complication in the other group $(\mathrm{p}=$ 0.372). Both groups had the same range of hospital stay after the operation (2 days). Superficial surgical site infection was diagnosed in one case $(10 \%)$ in each group, and they were managed by antibiotics in both cases. Transient post-operative hypocalcemia was encountered in 3 cases $(30 \%)$ in group A and 2 cases $(20 \%)$ in group $B(p=0.257)$.

Post-operative seroma was detected in 2 cases $(20 \%)$ in group A whereas only 1 case (10\%) in the other group had the same complication $(\mathrm{p}=0.248)$. Table (3) illustrates these data.

Table (3) Post-operative and follow up data.

\begin{tabular}{lccc}
\hline & Group A (n = 10) & Group B (n = 10) & P value \\
\hline Pain score (0 - 10) & $3.36 \pm 0.72$ & $2.97 \pm 0.62$ & 0.097 \\
Hospital stay (day) & $2(2-3)$ & $2(2-3)$ & 1 \\
Transient Hypocalcemia & $3(30 \%)$ & $2(20 \%)$ & 0.257 \\
Hematoma & $1(10 \%)$ & $0(0 \%)$ & 0.372 \\
Seroma & $2(20 \%)$ & $1(10 \%)$ & 0.248 \\
Superficial SSI & $1(10 \%)$ & $1(10 \%)$ & 1 \\
\hline
\end{tabular}

\section{Discussion}

So as to evade danger of complexities in all out thyroidectomy, accentuation is given to intensive analyzation for exact ID of anatomical structures and precise hemostasis [8].
Molnar et al. detailed their perception that utilizing the LigaSure little jaw gadget to perform absolute thyroidectomy is a dependable choice [9].

In late investigations viability of LigaSure in hemostasis and analyzation has been exhibited in 
thyroidectomies. Notwithstanding, the impact of LigaSure use in diminishing activity time, medical clinic remain, and intraoperative and postoperative inconveniences are questionable [10].

Ahmed et al., indicated that there was no factually huge contrasts among age and sex with respect to the traditional and ligasure gatherings [11].

Schiphorst et al., remembered 18 females and 2 guys for the customary gathering, while the ligaSure bunch included 17 females and 3 guys. They identified no critical distinction between the two gatherings $(p>0.05)$ [12].

Ramouz and his partners revealed that MNG was the commonest sign of thyroidectomy in their examination (43.4\% and $52.5 \%$ individually), trailed by papillary injuries (26.6 and $24.9 \%$ separately). Other reason included follicular sores, Grave ailment, thyroid adenoma, Hashimoto

In Ramouz et al., study, there was no factually noteworthy contrast between the two gatherings with respect to activity type absolute thyroidectomy or hemithyroidectomy in the two gatherings $(p=0.24)$ [13].

Hirunwiwatkul et al., revealed a usable time of $62.4 \pm$ 15.9 minutes in LigaSure bunch versus $83.3 \pm 16.1$ minutes with traditional strategy with a measurably huge contrast $(\mathrm{p}<0.001)[8]$.

Likewise, Molnar et al revealed a decrease in employable time from $80 \pm 12.4$ minutes when thyroidectomy was performed with the assistance of LigaSure Small Jaw versus a usable time of $106 \pm 23.5$ minutes for ordinary thyroidectomy $(\mathrm{p}=0.006)$ [9].

Moreover, Bhettani et al., indicated that the usable time was essentially drawn out in the ordinary gathering (123 versus 92 minutes in the ligaSure gathering $-\mathrm{p}<$ $0.01)[14]$.

A meta-examination by Zhang et al. likewise detailed huge decrease in employable time in LigaSure bunch versus ordinary stitch ligation strategy $(\mathrm{p}<0.00001)[11$, $15]$.

Bhettani and his partners likewise revealed that perpetual RLN paralysis didn't happen in their examination. Nonetheless, transient paralysis was experienced in 1.9 and $0.9 \%$ of cases on the two gatherings separately [14].

In an investigation by Khafagy et al, hematoma arrangement happened in 1 out of 15 patients $(6.7 \%)$ in traditional gathering versus none in the LigaSure little jaw gathering $(\mathrm{p}=0.098)(16)$.

Thus, the meta-investigation by Zhang et al didn't report measurably critical distinction in hematoma arrangement between the two gatherings individually $(\mathrm{p}=0.94)$ [15].

In Ahmed et al., study demonstrated the mean postoperative blood misfortune was likewise seen as fundamentally decreased in Ligasure gathering (53.80 \pm $3.17 \mathrm{ml})$ versus customary technique $(63.80 \pm 3.31 \mathrm{ml})$ with $\mathrm{p}=0.001[11]$.

Al Juraibi et al., likewise revealed comparative postusable blood loss of $54.16 \pm 9.21 \mathrm{ml}$ with LigaSure versus $66.28 \pm 8.99 \mathrm{ml}$ with customary stitch ligation procedure individually $(\mathrm{p}<0.0001)$ [17].

Unexpectedly, the investigation by Coiro et al revealed nonsignificant post-employable blood misfortune among the two gatherings $(\mathrm{p}=0.105)$. The post-usable blood was $39.80 \pm 20.50 \mathrm{ml}$ in LigaSure little jaw bunch versus $45.37 \pm 21.37 \mathrm{ml}$ in regular strategy gathering [18]

Another investigation announced that the ligaSure bunch indicated essentially lower torment VAS scores all through the initial three postoperative days [16].

In accordance with these discoveries, Pons et al. revealed that the mean postoperative paracetamol fulfillment in patients who had traditional thyroidectomy was $1.4 \mathrm{~g}$ more noteworthy than those remembered for the ligasure bunch with a critical distinction for ligasure [19].

Others guaranteed that a diminished necessity for parallel skin withdrawal and the decrease in cut length in the LigaSure bunch most likely diminished the postoperative agony [20].

In another examination, hypocalcemia was experienced in 6.8 and $2.9 \%$ of cases in ordinary and ligaSure bunches separately. There was a huge increment in that entanglement in the customary gathering ( $\mathrm{p}<$ $0.05)$ [14].

Ramouz et al., randomized controlled preliminary outcomes demonstrated a fundamentally higher commonness of subclinical and clinical hypocalcemia after traditional thyroidectomy[13].

Different outcomes indicated that general frequency of seroma development in patients experienced thyroidectomy by ligaSure Small Jaw was 2.2\% [21], in any case, thinking about past examinations, seroma rate ensuing to customary thyroidectomy has been accounted for to be around 5\% [22].

Another investigation affirmed our discoveries as the length of medical clinic stay didn't contrast between the two gatherings $(\mathrm{p}>0.05)$. the mean term was 1.3 and 1.4 days for the traditional and ligaSure bunches individually [23].

Anyway in another examination, creators revealed that the clinic stay was altogether drawn out in the traditional gathering (3.2 days) contrasted with the ligaSure gathering (1.8 days) $(\mathrm{p}<0.05)$ [14].

Postoperative injury disease was watched somewhat higher after complete thyroidectomy than lobectomy because of more tissue injury (24) and the careful site contamination rate was recognized in the traditional stitch tie tying method gathering and there was no disease sign in the ligasure bunch in another investigation [25].

\section{Conclusion}

Based on our study results, it is evident that ligaSure is not only a safe method for hemostasis during thyroid surgery like the conventional procedure, but it also helps to perform the surgery in a significantly shorter operative time. 


\section{References}

[1] G.V.Walls, R.Mihai,Thyroid Gland Embryology, Anatomy, and Physiology. Endocrine Surgery in Children: Springer, Vol.6, PP.3-15,2018.

[2] M.Ali,M.Murtaza,M.Aleem-ud-Din, Comparison of Harmonic Scalpel versus conventional hemostasis in Thyroid surgery in terms of per-operative and postoperative outcome. The Professional Medical J., Vol.4, PP.68-73,2020.

[3] L.Revelli,G.Damiani,CBNA.Bianchi,Complications in thyroid surgery. Harmonic Scalpel, Harmonic Focus versus Conventional Hemostasis: a metaanalysis. International J. Surgery., Vol.3, PP.S22S32,2016.

[4] G. Dionigi,W. Wu C, H.Y. Kim, Safety of energy based devices for hemostasis in thyroid surgery. Gland surgery., Vol.2, PP.29-32,2016.

[5] T.Youssef,T.Mahdy, M .Farid,Thyroid surgery: use of the LigaSure Vessel Sealing System versus conventional knot tying. international J. surgery, Vol.1, PP.42-62,2008.

[6] A.Y.Hammad, A.Deniwar, H. E .Mohamed, A prospective study comparing the efficacy and surgical outcomes of harmonic focus scalpel versus ligasure small jaw in thyroid and parathyroid surgery. Surgical innovation, Vol.4, PP.32-45,2016.

[7] N.Crichton. (VAS)Visual analogue scale. J.Clin Nurs, Vol.6, PP.7-21,2001.

[8] P.Hirunwiwatkul,S.Tungkavivachagul,A.multicenter .controlled clinical trial of LigaSure small jaw vessel sealing system versus conventional technique in thyroidectomy. European Archives of Oto-RhinoLaryngology, Vol.3, PP.14-19,2013.

[9] C.Molnar,S.Voidazan,C.Rad, Total thyroidectomy with LigaSure Small Jaw versus conventional thyroidectomy - a clinical study. Chirurgia (Bucur), Vol.3, PP.19-29,2014.

[10] G.Demiral, F. Aksoy,Comparison of ligasure and the conventional method in total thyroidectomy: a single surgeon's experience and review of the literature that ligasure is not superior to suture ligation in total thyroidectomy. THE ULUTAS MEDICAL J, Vol.3, PP.77-83,2019.

[11] W.Ahmed,T.Ibrahim,K.Ibrahim, COMPARISON BETWEEN TOTAL THYROIDECTOMY WITH LIGASURE SMALL JAW DEVICE VERSUS CONVENTIONAL VASCULAR LIGATURE TECHNIQUE. Pakistan Armed Forces Medical J, Vol.4, PP.91-95,2019.

[12] A.H.Schiphorst,B.A.Twigt,S.G.Elias, Randomized clinical trial of LigaSure versus conventional suture ligation in thyroid surgery. Head \& neck oncology, Vol.5, PP.13-25,2012.

[13] A.Ramouz,S.Z.Rasihashemi,A.Safaeiyan, Comparing postoperative complication of LigaSure
Small Jaw instrument with clamp and tie method in thyroidectomy patients: a randomized controlled trial [IRCT2014010516077N1]. World J., surgical oncology, Vol.3, PP.150-155,2018.

[14] M.K.Bhettani,M.Rehman,M.SKhan,Safety and Cost-effectiveness of LigaSure in Total Thyroidectomy in Comparison with Conventional Suture Tie Technique. Cureus, Vol.1, PP.3-19,2019.

[15]L.Zhang, Li N,X.Yang, A meta-analysis comparing the outcomes of LigaSure Small Jaw versus clampand-tie technique or Harmonic Focus Scalpel in thyroidectomy. Medicine, Vol.4, PP.155-170,2017.

[16] A.H.Khafagy,I.Abdelnaby,Total thyroidectomy: Ligasure versus Clamp \& Knot technique for intraoperative hemostasis. Egyptian J., Ear, Nose, Throat and Allied Sciences, Vol.7, PP.59-65,2013.

[17] W.AlJuraibi,R.Mohamed,A.Saber, Use of Ligasure Sealing Versus Conventional Suture-Ligation in Total Thyroidectomy. J Surg, Vol.2, PP.44-57,2016.

[18] S.Coiro, Frattaroli F, F.De Lucia.A comparison of the outcome using Ligasure ${ }^{\mathrm{TM}}$ small jaw and clampand-tie technique in thyroidectomy: a randomized single center study. Langenbeck's archives of surgery, Vol.3, PP.247-252,2015.

[19] Y.Pons,J.Gauthier,E.Ukkola-Pons, Comparison of LigaSure vessel sealing system, harmonic scalpel, and conventional hemostasis in total thyroidectomy. Otolaryngology-Head and Neck Surgery, Vol.4, PP.496-501,2009.

[20] S.V.Stager.Vocal fold paresis: etiology, clinical diagnosis and clinical management. Current opinion in otolaryngology \& head and neck surgery, Vol.5, PP.540-583,2014.

[21] A.Ramouz,S.Z.Rasihashemi,F.Daghigh.Predisposin $\mathrm{g}$ factors for seroma formation in patients undergoing thyroidectomy: Cross-sectional study. Annals of medicine and surgery, Vol.4, PP.330$350,2017$.

[22] A.Sanabria,A.L.Carvalho,C.E.Silver, Routine drainage after thyroid surgery-A meta-analysis. J., surgical oncology, Vol.1, PP.273-280,2007.

[23] T.Kirdak,N.Korun,H.Ozguc,Use of ligasure in thyroidectomy procedures: results of a prospective comparative study. World J. surgery, Vol.4, PP.170$190,2005$.

[24] G.Dionigi, F.Rovera,L.Boni, Surveillance of surgical site infections after thyroidectomy in a oneday surgery setting. International J.Surgery, Vol.2, PP.28-46,2008.

[25]B.Çakabay,M.M.Sevinç,Í.Gömceli, LigaSure versus clamp-and-tie in thyroidectomy: a single-center experience. Advances in therapy, Vol.1, PP.354,2009. 